\title{
Diffuse Astrocytoma, IDH-Mutant
}

National Cancer Institute

\section{Source}

National Cancer Institute. Diffuse Astrocytoma, IDH-Mutant. NCI Thesaurus. Code C129271.

Diffuse astrocytoma carrying IDH mutations. 